\title{
Underwater operation of a DBD plasma jet
}

\author{
John E Foster, Brandon Weatherford, Eric Gillman and Benjamin Yee \\ Department of Nuclear Engineering and Radiological Sciences, The University of Michigan, Ann Arbor, \\ MI, USA
}

Received 26 April 2009, in final form 15 December 2009

Published 25 January 2010

Online at stacks.iop.org/PSST/19/025001

\begin{abstract}
A plasma jet produced in water using a submerged ac excited electrode in a coaxial dielectric barrier discharge configuration was studied. Plasma jet formation was found to occur only while the source was submerged. Plasma jet operation was characterized with and without gas flow. It was found that over $60 \%$ of the discharge power was deposited into the water and did not vary appreciably with excitation frequency. Presumably the remaining power fraction went into excitation, ionization and local electrode heating. Emission spectra of the jet revealed nitrogen, hydrogen, hydroxyl and oxygen emission lines. Operation of the plasma jet in water containing the oxidation-reduction indicator methylene blue dye resulted in a marked clearing of the water as observed visually and with a spectrophotometer, suggesting plasma-induced chemical reactivity.
\end{abstract}

(Some figures in this article are in colour only in the electronic version)

\section{Introduction}

The use of nonthermal, gas phase atmospheric pressure plasmas in industrial processing is widespread with applications ranging from ozone production to the surface treatment of polymers and the like [1]. Another potential application of nonthermal plasmas involves those chemical processing reactions that utilize liquids such as water as either a reagent or a solvent. Liquid water is a ubiquitous and universal solvent used extensively in chemical processing [2]. Currently nonthermal plasmas are being researched for the treatment of dye-contaminated water produced in textile plants as well as for the general purification of drinking water [3-7]. Many of these efforts feature plasma surface treatment of the water, which in turn produces reactive radicals that ultimately oxidize the contaminant molecules in the water $[8,9]$.

In the work presented here, we investigate the operation of an air-driven dielectric barrier discharge jet that could potentially be used to affect the chemical reactivity of liquid water. Unlike conventional atmospheric pressure plasma jets, this plasma source actually operates submerged underwater, offering the prospect of in-volume treatment [10-12]. The general operation of the plasma jet in water is presented and discussed. A preliminary assessment of the plasma jet's effectiveness in treating methylene blue (MB) dye in dionized water is also discussed.

\section{Experimental set-up}

A schematic depiction of the apparatus used in this investigation is shown in figure 1 .

The discharge device consisted of a cylindrical, copper electrode coaxially fed into a $5 \mathrm{~mm}$ OD, $2.5 \mathrm{~mm}$ ID ceramic tube. The ground electrode consisted of a coil of tantalum wire wrapped about the end of the aluminum oxide tube. The external coil was insulated from the water using a kapton shield not shown in the figure. Figure $1(b)$ illustrates the equivalent circuit. Here, $C_{1}$ represents the effective capacitance of the central electrode to ground through the intervening material which consists of the ceramic tubing, the air cavity produced just below the exit plane of the tube during operation, and the water. $\mathrm{C}_{2}$ represents the capacitance between the central electrode and outer coupling coil, illustrated in figure $1(a)$. Air was fed into the tubing as depicted in the figure using a compact air compressor. The air compressor flow was measured using an inline airflowrotameter with a readout uncertainty of $\sim 0.1 \mathrm{~L} \mathrm{~min}^{-1}$. Unless stated otherwise, for all air injection experiments described here, the airflow was adjusted to $1.9 \mathrm{~L} \mathrm{~min}^{-1}$. In the tests described herein, the discharge tube was submerged into a $100 \mathrm{~mL}$ graduated cylinder containing deionized water with a specific conductance of $0.0714 \mu \mathrm{S} \mathrm{cm}^{-1}$. Figure 2(a) depicts source operation with airflow but no applied voltage. Air 


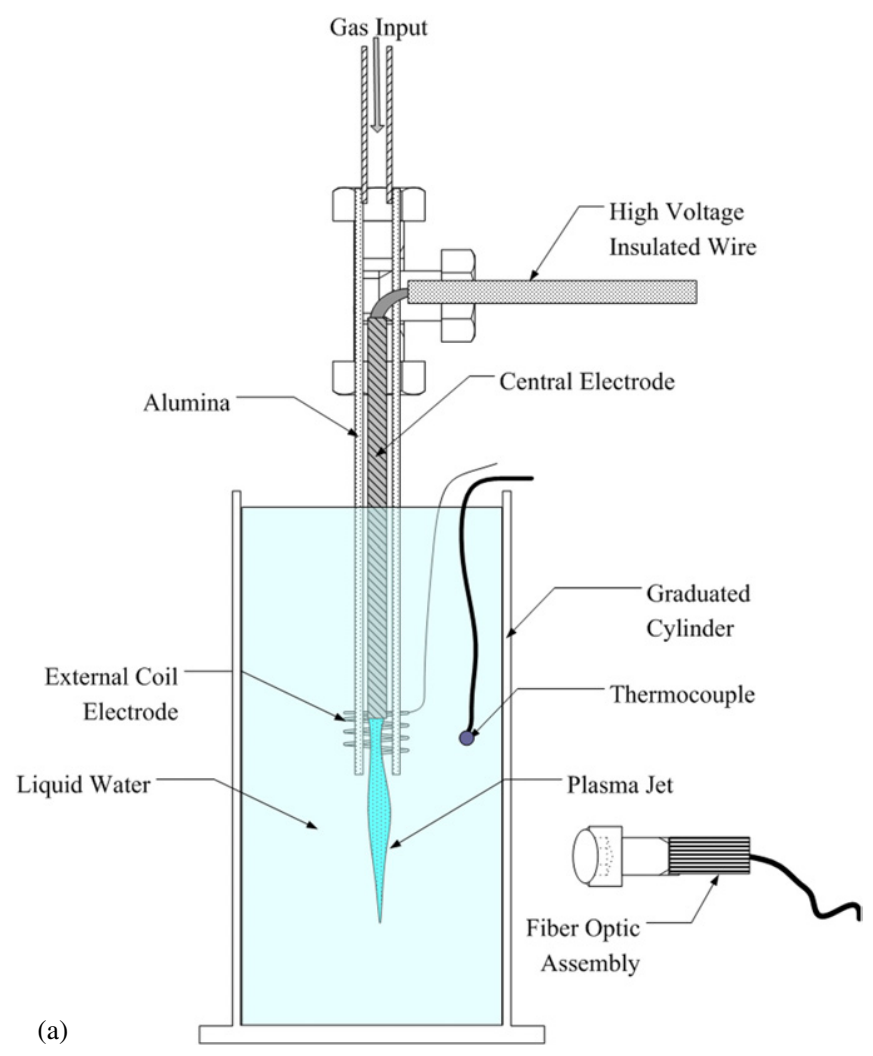

(a)

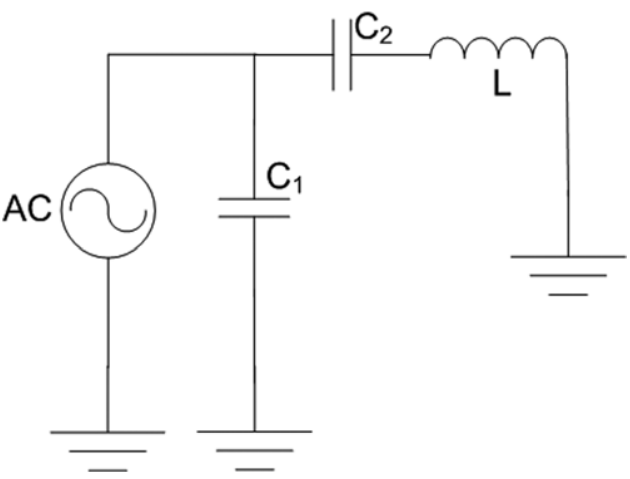

(b)

Figure 1. (a) Schematic depiction of the apparatus used to produce the plasma jet and characterize its effect on liquid water. $(b)$ Equivalent circuit depiction of the apparatus.

injection into the water below the ceramic tube as well as bubble break-off leading to the formation of a multitude of smaller bubbles may be seen in the photograph. It should be pointed out that the method of gas injection utilized in this experiment is not true bubbling, rather it is best characterized as water agitation by direct injection of gas. The forced air forms an unstable cavity or pocket in the water. The cavity is inherently unstable, giving rise to breaking and subsequent bubble formation. The plasma jet resides in this cavity.

In all cases, the injected airflow essentially stagnated after penetrating the water and then turned upward under buoyancy forces as shown in the photograph. This general behavior was unaffected by the presence of the plasma jet. The central electrode was coupled to the variable frequency ac source (0-200 V output) through a high voltage transformer $(10 \mathrm{kV}, 1: 500)$, represented simply as the ac source depicted in figure $1(b)$. The frequency range investigated in this work

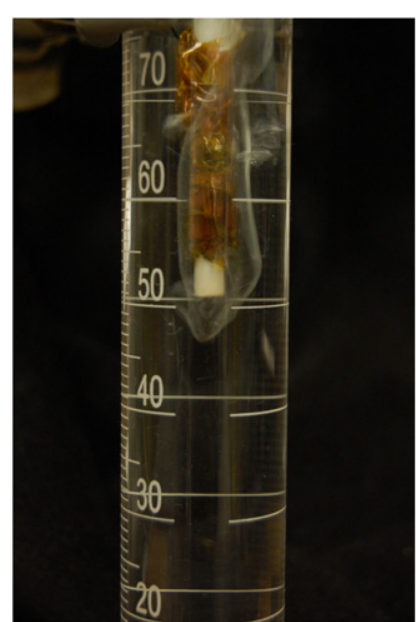

(a)

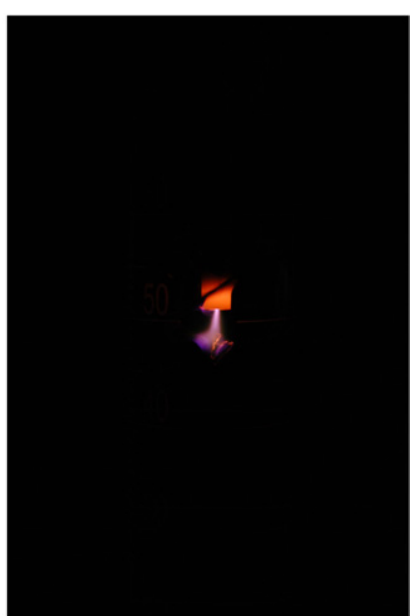

(b)
Figure 2. Photograph of the discharge tube with airflow only $(a)$ and with airflow and discharge active $(b)$. Note the plasma jet does not terminate at an external electrode. (Discharge: $1 \mathrm{kHz}, 2500 \mathrm{~V}$ amplitude, $1.9 \mathrm{~L} \mathrm{~min}^{-1}$.)

extended from 1 to $4 \mathrm{kHz}$. Plasma-induced water temperature changes were monitored as a function of time using in situ, submerged type $\mathrm{K}$ thermocouples. The current was measured using a Pearson coil rated at up to $120 \mathrm{MHz}$ with a usable rise time of $5 \mathrm{~ns}$. The voltage was measured using a Tektronics 1000: 1 high voltage probe. The signals from the probes were monitored using a $60 \mathrm{MHz}$ digital oscilloscope, which allowed for the resolution of signals structures down to $100 \mathrm{~ns}$. A photograph of the discharge operating with airflow, submerged in deionized water is depicted in figure $2(b)$. Plasma jet length was roughly $1 \mathrm{~cm}$. Orange glow of filtered light through the alumina tubing is also apparent.

Discharge initiation and nominal operation occurred while the discharge tube was submerged underwater. During discharge operation an Ocean Optics USB2000 spectrometer with a $25 \mu \mathrm{m}$ slit was used to assess species content in the plasma discharge, which emanated from the discharge tube as depicted in figure $2(b)$. The spectrometer's usable range extended from 200 to $1100 \mathrm{~nm}$, making near ultraviolet $\mathrm{OH}$ lines detectable. Spectrometer resolution is approximately $1.4 \mathrm{~nm}$.

\section{Experimental results}

The following sections describe results from measurements made during plasma jet operation. Section 3.1 of the results section discusses plasma jet formation, structure and behavior. Section 3.2 discusses the effect of the plasma jet on the deionized water, which includes water heating rates and power deposition. Section 3.2. also details source operation without gas flow. Section 3.3 briefly details results of the effect of the plasma jet on dye-laden deionized water.

\subsection{Current voltage characteristics of the plasma jet}

Using a high voltage probe and the current probe, plasma jet current-voltage (IV) variations with time were recorded. 

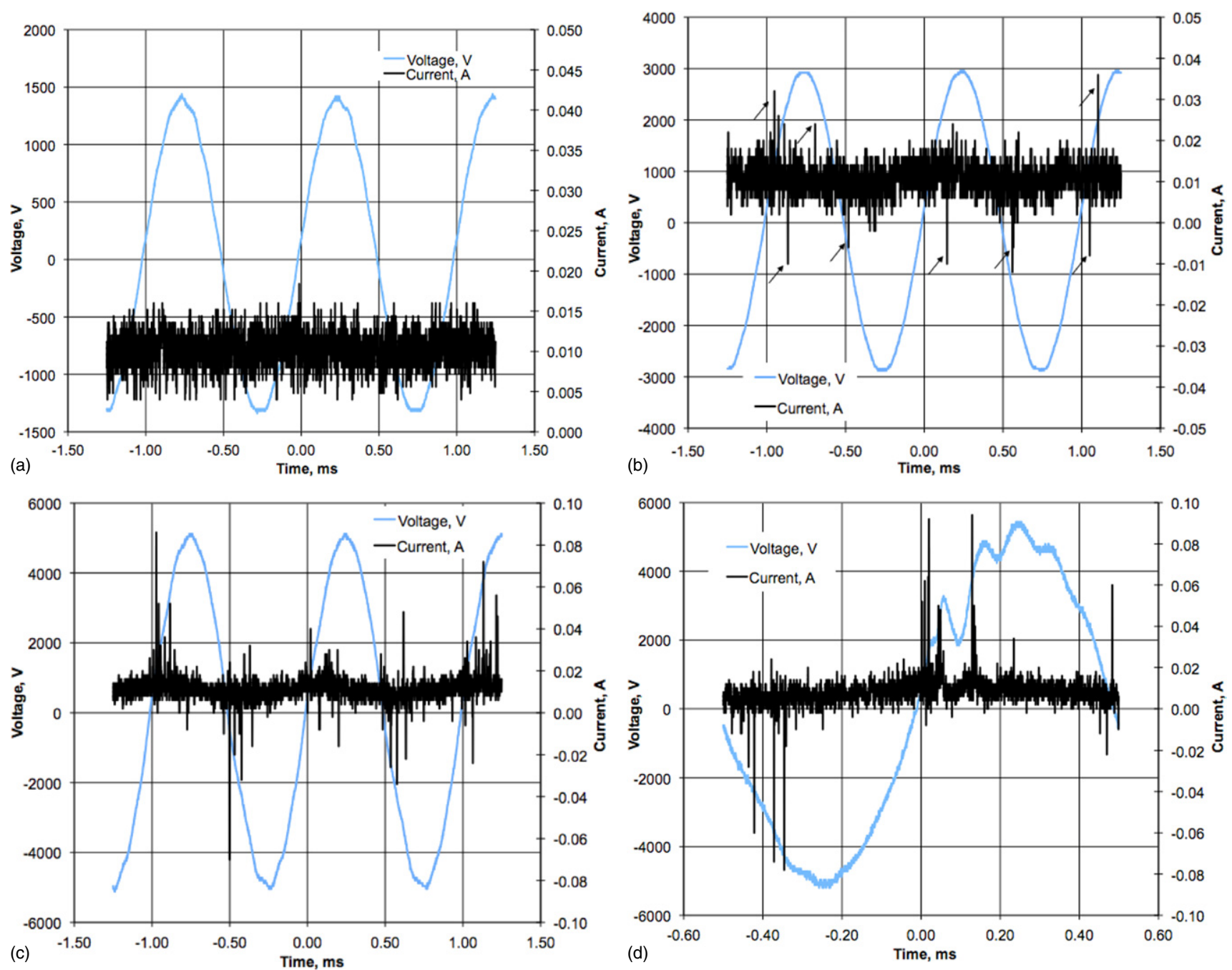

Figure 3. (a) At low peak-to-peak voltages, no breakdown activity is evident in the current waveform. (1 kHz, $1.9 \mathrm{~L} \mathrm{~min}{ }^{-1}$ airflow). (b) As indicated by arrows, the presence of microdischarges (spikes) is evident in the current waveform. $\left(1 \mathrm{kHz}, 1.9 \mathrm{~L} \mathrm{~min}^{-1}\right.$ airflow.) (c) Current spike intensity and number increase with increasing voltage. $\left(1 \mathrm{kHz}, 1.9 \mathrm{~L} \mathrm{~min}^{-1}\right.$.) $(d)$ Close up of apparent distortions to the applied voltage due to power supply loading just before emergence of the plume. (1 kHz, $1.9 \mathrm{~L} \mathrm{~min}^{-1}$ airflow.)

Typical $I V$ data acquired at $1 \mathrm{kHz}$ ac excitation frequency are presented in figure 3. Figure 3(a) illustrates the measured voltage and current response at an applied peak-to-peak voltage $(2.86 \mathrm{kV})$ just below that required for the appearance of microdischarge current spikes. The current signal measured here is assumed to be associated with noise. A weak ac modulation is also suggested in the figure. This modulation is attributed to finite conductivity of the water. Increasing the voltage to just above $3 \mathrm{kV}$ peak-to-peak gave rise to the appearance of current spikes (widths $<1 \mu \mathrm{s}$ ) which were assumed to be associated with microdischarge formation.

Increasing the peak-to-peak voltage level to $6 \mathrm{kV}$ gave rise to the appearance of more discharge current spikes as indicated in figure $3(b)$. The locations of the current spikes are indicated by arrows. The current spikes on average appear to alternate in sign sequentially with each breakdown suggesting the residual charge effect typical of barrier discharges [13, 14]. Despite the presence of the current spikes, the presence of a discharge external to the tube was not observable. Later internal measurements indicated that the microdischarges were actually localized to the interior of the tube.
Further increase in the voltage to $10.6 \mathrm{kV}$ peak-topeak revealed a significant increase in discharge activity as illustrated in figure $3(c)$. At this voltage level, the amplitude of the current spikes increased appreciably, actually doubling in the more extreme cases. Significant modulation of the waveform is also observed. The increased amplitude and number of current spikes indicate intensification of the microdischarges within the tube. A rise in water temperature at this operating point of order $1^{\circ}$ was also observed.

A slight increase in peak-to-peak voltage above $10.6 \mathrm{kV}$ resulted in severe distortion of the voltage waveform, presumably due to a combination of power limitations of the power supply (400 W average power) and significant impedance drop in the load. To better illustrate the distortions as well as acquire a more stable waveform, the time base was adjusted to show only one cycle. These distortions are illustrated in figure $3(d)$, which are dubbed 'sub-plume threshold' as voltages above this value resulted in the emergence of the plasma jet. Figure $3(d)$ illustrates the $10.8 \mathrm{kV}$ peak-to-peak condition. As can be seen here, the current spikes occur at those instances where the time derivative 
of the voltage is greatest. At the sub-plume threshold condition, it was difficult to quantify the heating rate. The discharge tended to transition spontaneously into the plume mode with the emergence of the plasma jet if left at this voltage condition for an appreciable time. This behavior may be associated with electrode heating and subsequent thermionic emission. Figure 4(a) illustrates the associated waveform just beyond sub-plume threshold. The current profile is completely modulated, behaving more arc-like rather than a streamer. Such arc-like modes have been observed in both atmospheric pressure glow discharges and water plasma discharges [15-17]. In the case of the atmospheric glow discharge, at sufficiently high voltage, an arc mode appears often with the simultaneous appearance of microdischarge current spikes [16]. Microdischarge current spikes are also present on the waveform during the arc-like mode observed in this work as well. The modulation of the current suggests real current effects as there is no appreciable phase shift between the current and voltage signal as would be expected from displacement current effects. The mechanism for the appearance of the arc-like mode and its associated bipolar current is not well understood, though electrode processes likely play a significant role as inferred from observed melting of the central electrode and the drop in voltage once the plume emerged. Certainly the intensification of microdischarges will lead to localized heating. In the case of the appearance of the arc-like mode in the atmospheric pressure glow discharges, both electrodes are typically metal with cathode spots driving the transition [18]. In this study, the second electrode is separated by a dielectric barrier (water and alumina). Remarkably, once the plume emerges, the discharge behaves as an arc burning in the gas jet under water without any apparent return electrode. It should also be pointed out that the jet would preferentially make contact with a grounded electrode if such an electrode were to be placed nearby, indicating that the discharge was indeed an unterminated, propagating arc-like jet seeking ground and not simply exiting, excited gas.

Further increases in voltage gave rise to a significant drop in impedance as depicted in figure $4(b)$. Indeed, attempts to increase the voltage did not appear to have any appreciable effect on the discharge. In general, the discharge could not be initiated at the low voltages ( $<5000 \mathrm{~V}$ peak-to-peak) depicted in the figure, suggesting significant hysteresis and 'bootstrap' effects. The drop in voltage is suggestive of a fundamental discharge mode change. This behavior is akin to the sheath breakdown also observed in atmospheric pressure glow discharges during the transition to arc mode [16]. The time scales for the temporal distortions in the current and voltage profile are sufficiently large (ms) that features are resolvable, allowing for the calculation of real power associated with the discharge $[13,19]$. This is to be contrasted with power calculations made in conventional barrier discharges in which the current spikes are quite random and difficult to resolve from oscillograms $[19,20]$. Power deposition calculations are presented later in this work.

3.1.1. The plasma jet-optical characteristics. Figure 5 depicts the emission spectra acquired from the plasma jet. As
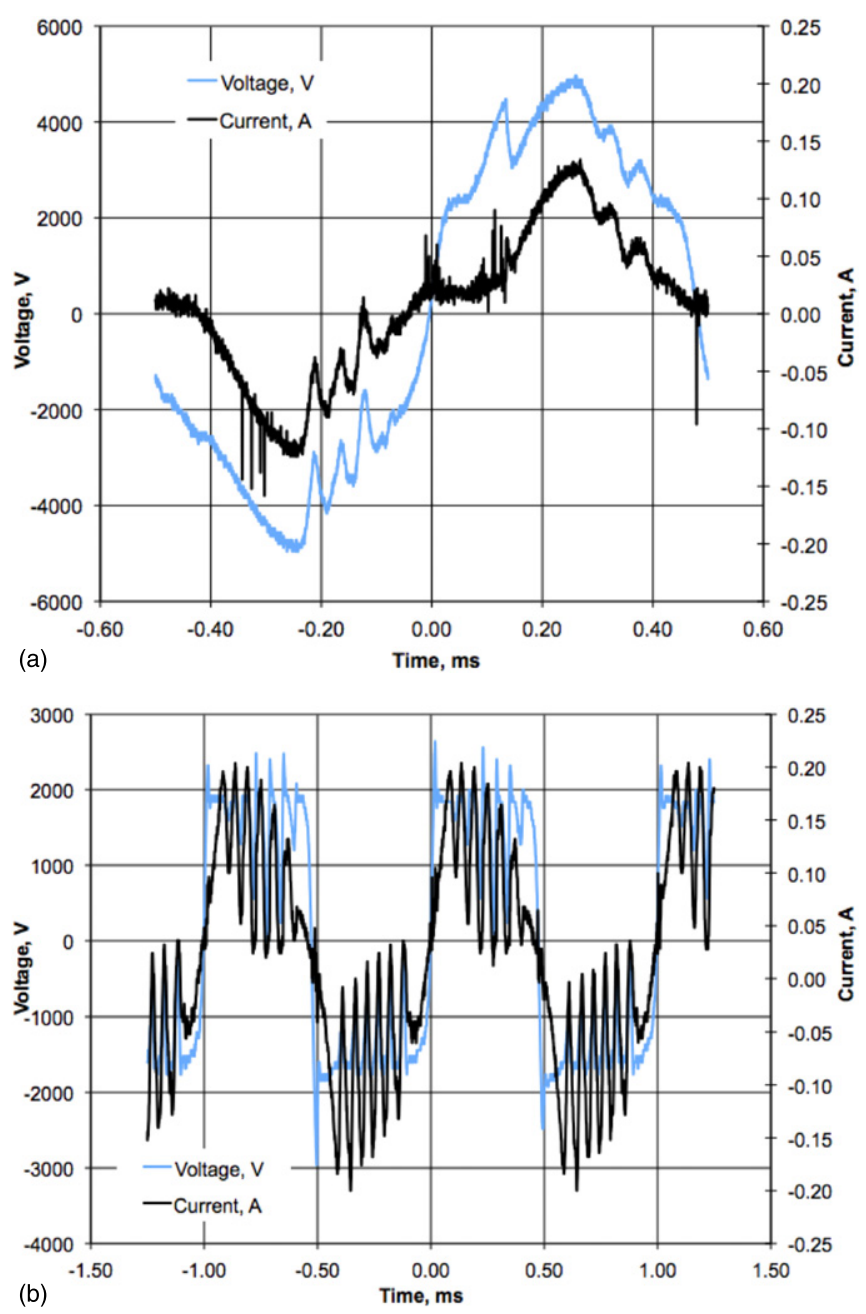

Figure 4. (a) Waveform appearance at the onset of plume appearance. The current waveform is completely modulated at the excitation frequency. Microdischarge spikes are also present.

$\left(1 \mathrm{kHz}, 1.9 \mathrm{~L} \mathrm{~min}^{-1}\right.$ airflow.) (b) A further increase in voltage gives rise to a significant drop in impedance and thus applied voltage as the current increases. $\left(1 \mathrm{kHz}, 1.9 \mathrm{~L} \mathrm{~min}^{-1}\right.$ airflow.)

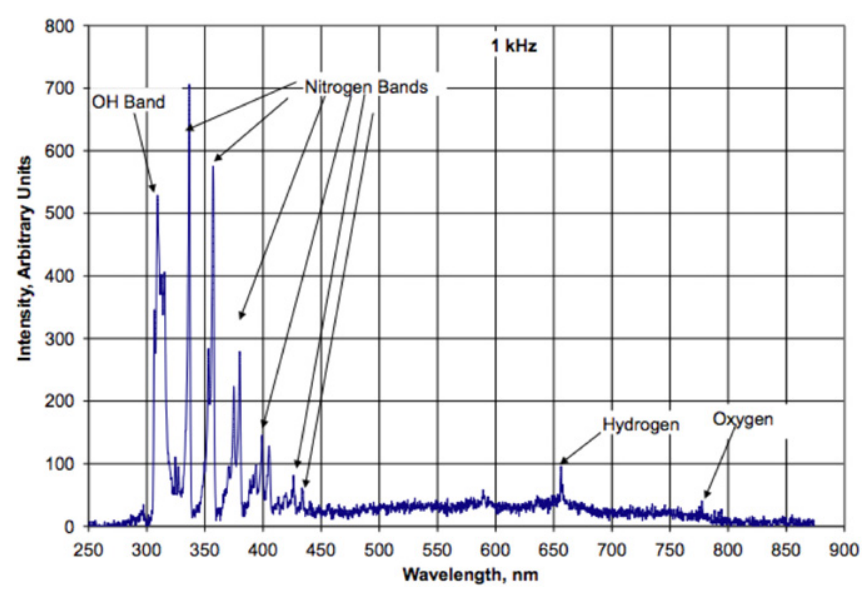

Figure 5. Emission collected from the plasma jet. Spectra are dominated by nitrogen and $\mathrm{OH}$ emission. Two copper lines centered around $325 \mathrm{~nm}$ are also apparent. (Discharge: $1 \mathrm{kHz}, 2500 \mathrm{~V}$ amplitude, $1.9 \mathrm{~L} \mathrm{~min}^{-1}$.) 


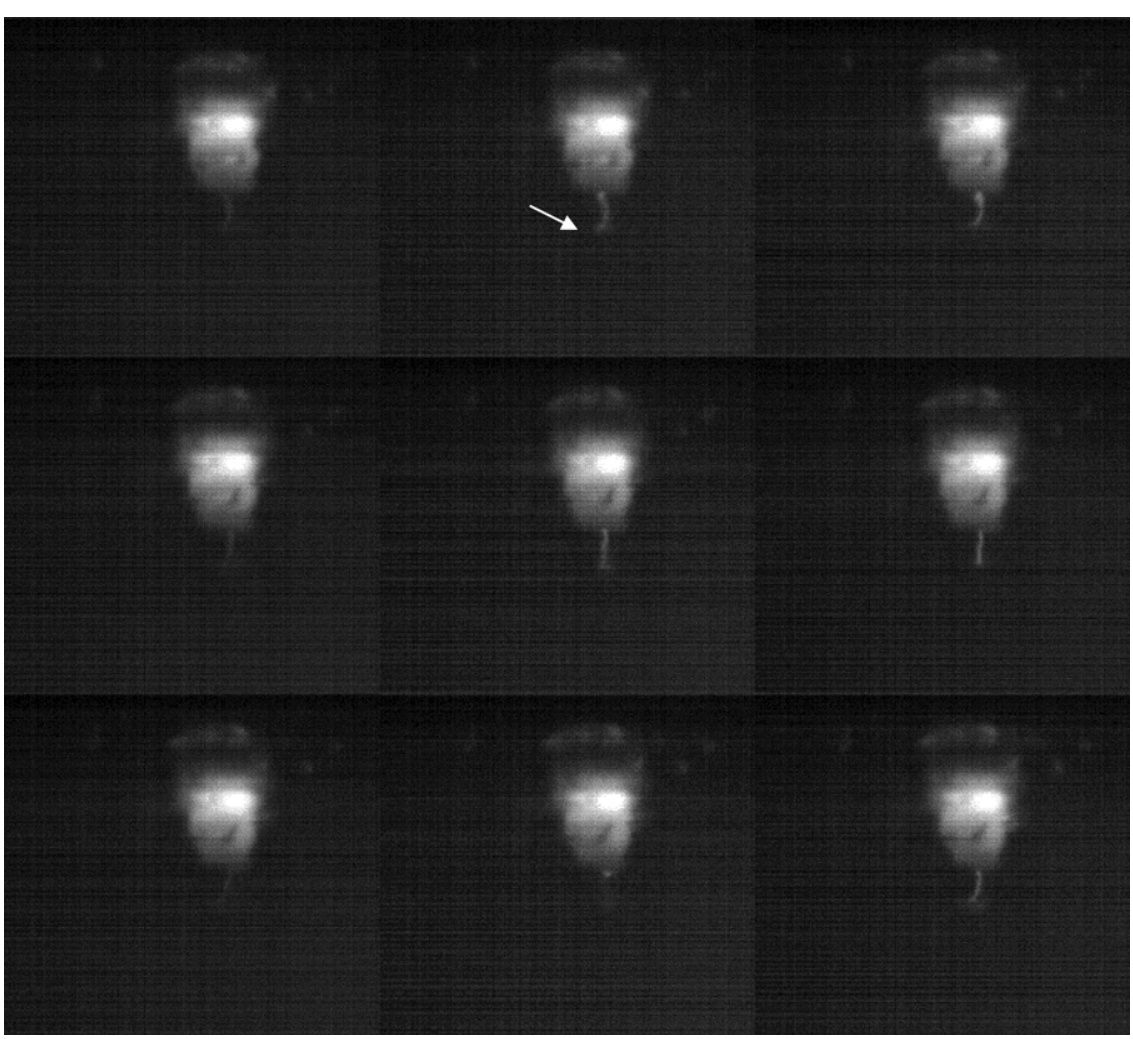

Figure 6. Snapshots of a single streamer (arrow) during discharge operation. Note the ropy structure emanating from the tube. Tube glow is also apparent indicating the presence of an intense discharge within. The camera acquisition rate is $1 \mathrm{kHz}$. (Discharge: $1 \mathrm{kHz}, 2500 \mathrm{~V}$ amplitude, $1.9 \mathrm{~L} \mathrm{~min}^{-1}$.)

can be seen here, the spectra are dominated by strong emission from nitrogen as reflected in the presence of second positive band system [21]. Also prominent is the $\mathrm{OH}$ emission band, which suggest nontrivial amounts of water vapor in the jet. Oxygen $(777 \mathrm{~nm})$ and the hydrogen alpha line at $656 \mathrm{~nm}$ are also apparent in the spectra. Careful inspection of the spectra also revealed two copper lines at 324 and $328 \mathrm{~nm}$ convoluted with the $\mathrm{OH}$ band. The presence of these lines is consistent with electrode melting indicated by the change in electrode surface texture observed post-test. The local heating of the electrode and subsequent vapor production are suggestive of arc-like behavior.

Once the jet emerges, the plume itself has the visual appearance of a rather stationary cylindrical ray emanating from the central electrode and projecting into the air-water mixing region below. Surrounding this central discharge is a luminous plume of presumably excited gas and plasma. The discharge resided exclusively within the jet of injected air. The injected flowing air penetrated the water for approximately $2 \mathrm{~cm}$ before stagnating and reflecting upward. Presumably at the stagnation boundary, the air jet pressure matches the water pressure at that level. In order to obtain more insight into the nature of this plume, a fast frame rate camera was used to capture the time varying behavior of the plasma jet. At 1000 frames s $^{-1}$, the camera gives time-averaged behavior over a cycle. At this acquisition rate, as indicated in figure 6 , it was found that the discharge was not stationary. In general when the frames are run in sequence, some insight into actual jet behavior can be gleaned. It was observed that the end of the discharge itself terminated at the water-bubble interface. The intensity and the position of the jet glow appear to vary from frame to frame suggesting different intervals of arc-like discharge were being sampled. These variations are consistent with variations in observed current with time discussed earlier.

\subsection{Discharge effects: sound and heat}

With the emergence of the plasma plume into the water, a noticeable change in sound emanating from the graduated cylinder as well as an expected rise in temperature were observed. Correlation between changes in discharge emitted sound and the onset of a discharge mode change has been observed in atmospheric pressure glow discharges [22]. The sound is a manifestation of time dependent heating of the injected air by the plasma jet current at the excitation frequency and harmonics. As can be seen from the current waveforms (figures 3-4), a plethora of harmonics should be possible. This was indeed the case as could be seen from a comparison of FFT sound signals with and without plasma present as shown in figure 7. The observed harmonics appear to alternate in intensity with on average the even harmonics having a lower intensity in comparison with odd harmonics.

Readily noticeable upon emergence of the plume was the rather rapid rise in water temperature with time. To further understand the temperature changes as well as the spatial variation in the temperature, thermocouple measurements were made at three locations: (1) at the plane of the plasma tube exhaust, (2) $\sim 1 \mathrm{~cm}$ from the bottom of the graduated cylinder 


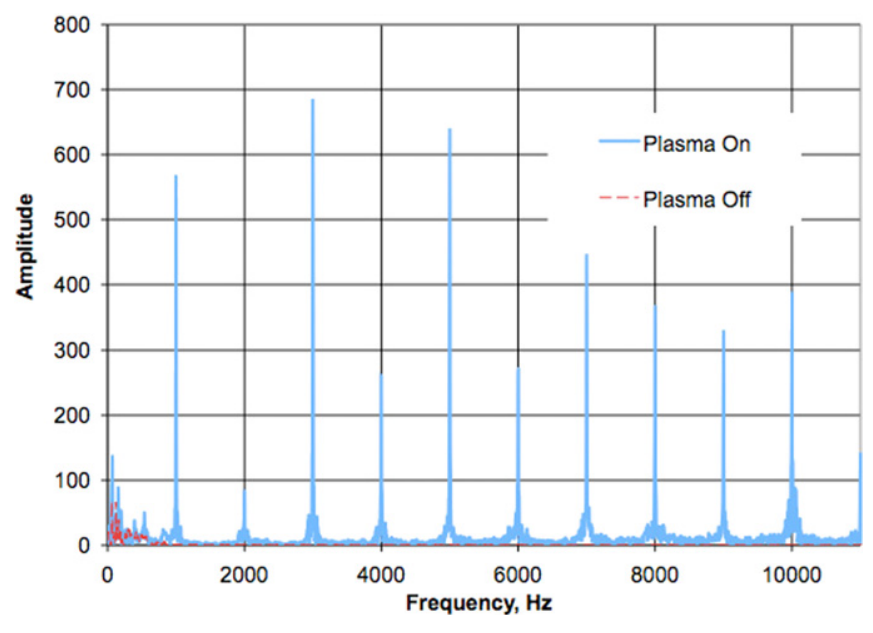

Figure 7. FFT of sound emitted from the graduated cylinder with and without plume formation. (Discharge: $1 \mathrm{kHz}, 2500 \mathrm{~V}$ amplitude, $1.9 \mathrm{~L} \mathrm{~min}^{-1}$.)

( $\sim 4 \mathrm{~cm}$ from the discharge tube exit) and (3) $1 \mathrm{~cm}$ below the water level of the graduated cylinder. These measurements were made to ascertain spatial variations in local heating effects associated with the localized plasma jet.

It was found that the water was heated anisotropically with the water temperature in the upper half of the graduated cylinder increasing much more rapidly than that of the lower half. The water temperature measured near the discharge tube exit was similar to that measured at the upper portion of the graduated cylinder. For the time intervals investigated in this work $(\sim$ few minutes $)$, the water in the lower portion of the cylinder (below $50 \mathrm{ml}$ line) remained essentially at room temperature giving rise to a situation where the lower density, hot water was localized to the top half of the graduated cylinder and the denser, room temperature water residing at bottom half. Also noticeable between the two halves of the water column (roughly above and below the $50 \mathrm{ml}$ line) were convection patterns associated with mixing. In this case, however, the hotter, lower density medium is already on top, making mixing via convection more laborious. Figure 8 illustrates this localization of water heating at an ac excitation frequency of $1 \mathrm{kHz}$ for the plasma source cases with and without airflow. The zero airflow case will be discussed later. Here, thermocouple A (TCA) measures water temperatures near the bottom of the column of water $(\sim 5 \mathrm{ml}$ fill line) while thermocouple B (TCB) measures temperatures near the top of the column ( $\sim 90 \mathrm{ml}$ fill line). This finding suggests that energy exchange is dominated by heat exchange between plasmaheated, buoyant heated air and the water. This mode of energy exchange is the likely cause for the anisotropic water heating at least at early times ( $t \sim$ few minutes).

Figure 9 illustrates the temporal behavior of approximately $100 \mathrm{ml}$ of deionized water as a function of time for four different excitation frequencies assessed from temperature measurements made near the tube exit with airflow. As can be seen from the data, the temperature rise is rapid and essentially linear. The similarity of slopes suggests that the heating rates are relatively insensitive to the frequency over the range investigated here.

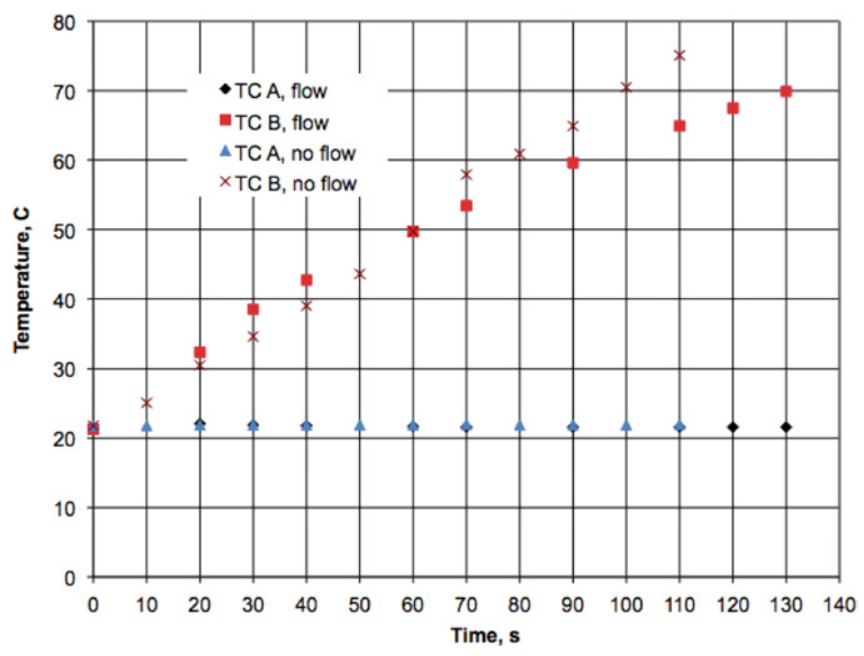

Figure 8. Heating rates at two locations within the water column. (Air discharge: $1 \mathrm{kHz}, 2500 \mathrm{~V}$ amplitude, $1.9 \mathrm{~L} \mathrm{~min}^{-1}$; no airflow.)

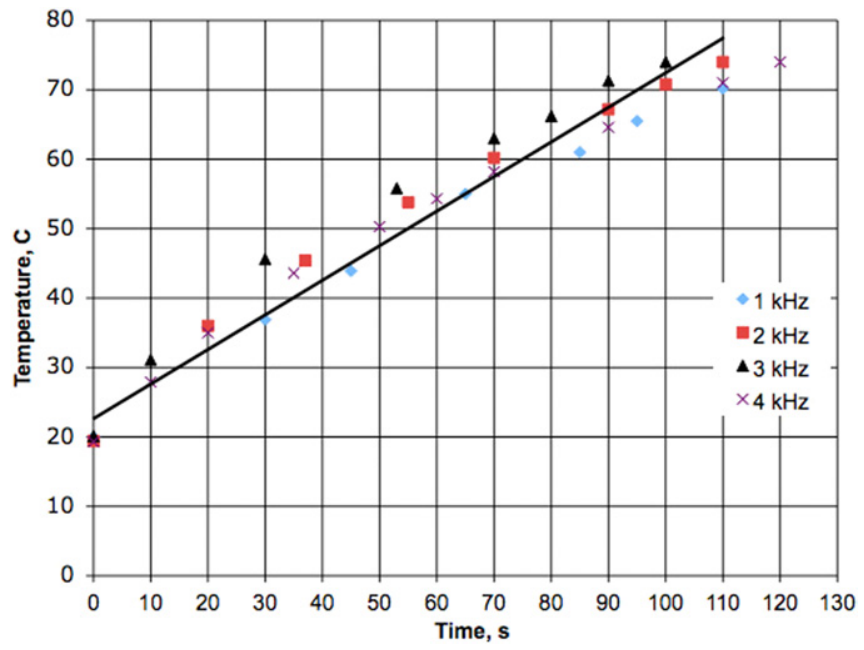

Figure 9. Variation in water heating rates with excitation frequency. (Discharge conditions: approximately $2500 \mathrm{~V}$ amplitude, 1.9 $\mathrm{L} \mathrm{min}^{-1}$.)

As mentioned earlier (see figure 8), the heat transfer was highly nonuniform with water below the jet not being heated appreciably and water above the jet receiving significant heating. In order to obtain a rough estimate of the power absorbed by the water, the discharge power deposited into the water was assumed to be localized to the water in the upper half of the graduated cylinder. In this case, it is assumed that the mass of water absorbing energy was primarily the upper $50 \mathrm{ml}$. This calculation should yield a reasonable estimate of power deposited into the water. The power absorbed by this water is simply

$$
P_{\text {water }}=\frac{m c_{v} \Delta T}{\Delta t}
$$

where $\Delta t$ is the total time the discharge is active and $\Delta T$ is the temperature change over this interval. This relation assumes that heating rate is constant, an assumption justified given the linear rise in temperature as a function of time. The water mass $m$ is also assumed roughly constant, a condition also satisfied in this work as the bulk water temperature was not allowed to exceed $80^{\circ} \mathrm{C}$. A rough estimate of the discharge 


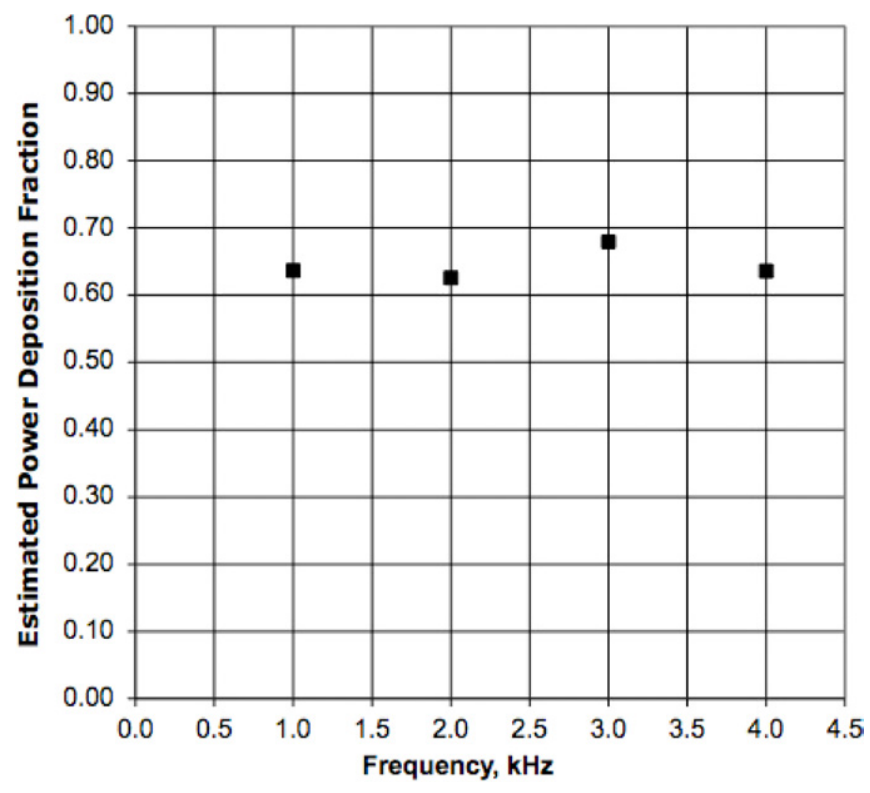

Figure 10. Variation in estimated power absorbed as a function of frequency. (Discharge conditions: approximately $2500 \mathrm{~V}$ amplitude, 1.9 $\mathrm{L} \mathrm{min}^{-1}$.)

power was estimated by numerically integrating the product of the current and voltage curve over a cycle to get the average real energy absorbed:

$$
E_{\text {discharge }}=\int_{0}^{2 \pi / \omega_{o}} I(t) \cdot V(t) \mathrm{d} t .
$$

The product of this parameter and the ac frequency yields the average power dissipated per cycle. Figure 10 illustrates the behavior of the ratio of estimated power absorbed by the upper portion of the graduated cylinder to the integrated discharge power.

As can be seen in the figure, the estimated power fraction is essentially flat, indicating that fraction discharge power that makes it into the water does not change appreciably with frequency. The relative insensitivity to frequency is consistent with liquid water heating rate, which did not vary appreciably with frequency. The remaining power fraction, $\sim 35 \%$, presumably goes into processes such as ionization, excitation and subsequent radiation. Additionally, some power is also deposited into the exposed central electrode, as evidenced by localized melting at its tip.

3.2.1. Operation without airflow. The source was also capable of operating underwater without any injected gas flow. Figure 11 illustrates the discharge without airflow operating at $4 \mathrm{kHz}$. As can be seen in the figure, a large bubble is formed at the exit of the source. The arc-like discharge is sustained in this bubble. In general, the discharge could be operated without airflow over the frequency range investigated here $(1-4 \mathrm{kHz})$. The discharge color, however, was quite sensitive to frequency range changing from predominantly green at $1 \mathrm{kHz}$ to mostly white with a green and violet tinge at $4 \mathrm{kHz}$. The bubble or cavity in which the discharge 'burns' is presumably composed of water vapor. Periodic breaking

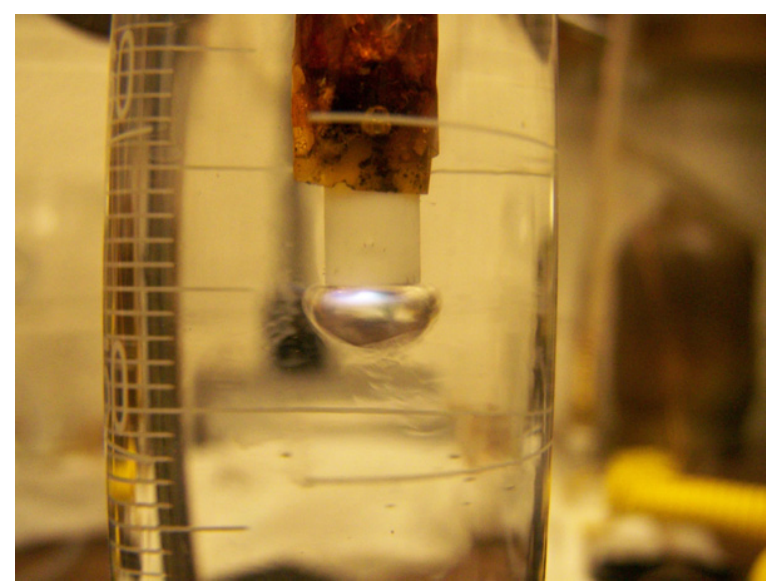

(a)

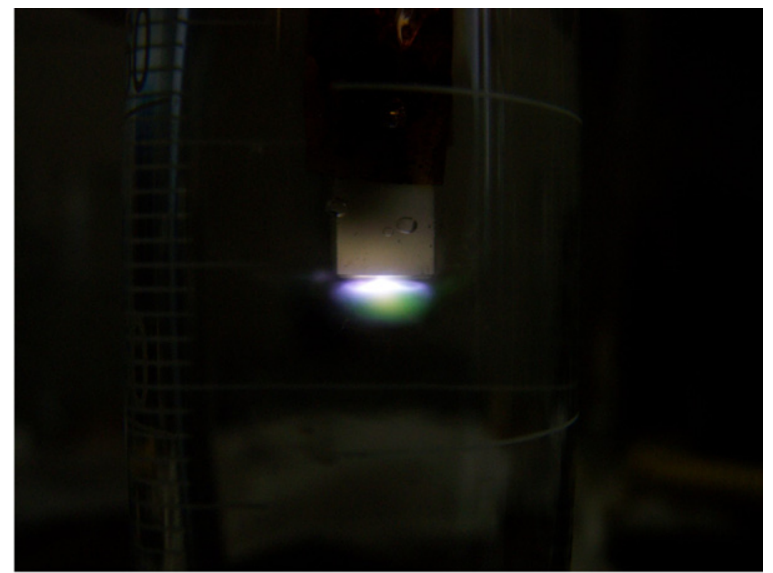

(b)

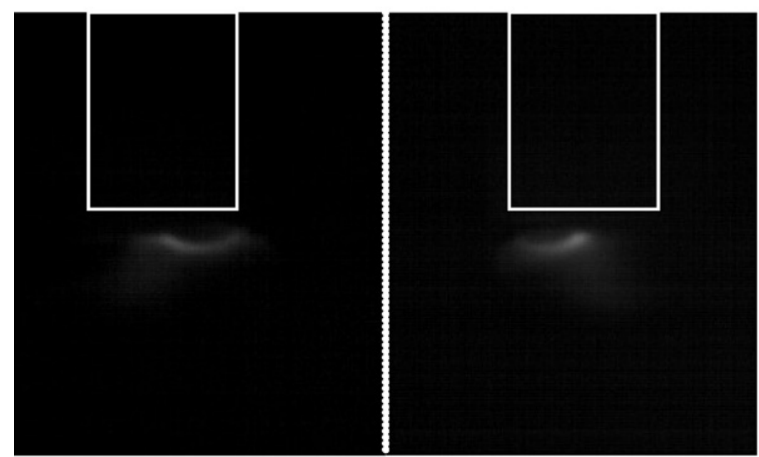

(c)

Figure 11. Discharge operation without airflow. (a) Acquired with flash, the steam bubble is observable. $(b)$ Acquired in a darkened room, plasma is apparent as well as small bubbles formed by breaking. (c) Highly curved streamers imaged using a high-speed camera. The white rectangle depicts the approximate outline of the alumina tube end. (Discharge: $4 \mathrm{kHz}, 2500 \mathrm{~V}$ amplitude, $1.9 \mathrm{~L} \mathrm{~min}^{-1}$.)

of the bubble due to instabilities and turbulence is a source of smaller bubbles as seen in figure 11(b). Also, noticeable just below the large bubble (figure 11( $a)$ ) is a change in the index of refraction of the water caused changes in water density brought on by the localized heating. These wispy, shimmering regions constitute a convection zone.

Based on high-speed camera images, the discharge, like the airflow case, consisted of a single jet that emanated from 


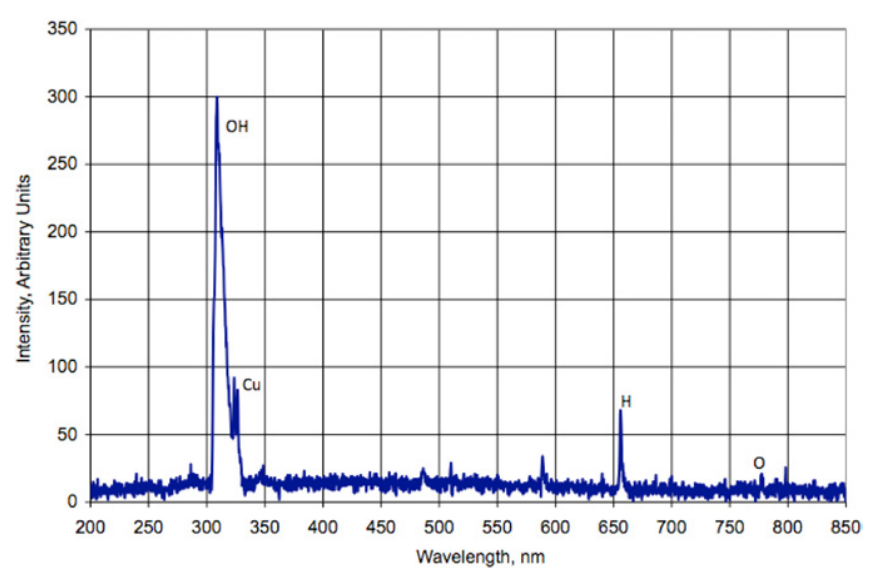

Figure 12. Emission spectra from the source operation without airflow. Hydrogen Balmer and $\mathrm{OH}$ band peaks are most prominent. (Discharge: $4 \mathrm{kHz}, 2500 \mathrm{~V}$ amplitude, $1.9 \mathrm{~L} \mathrm{~min}^{-1}$.)

the center of the discharge tube but differed in that it tended to curve toward the edge of the tube as shown in figure 11(c).

The key mechanism enabling operation without airflow is believed to be field emission-induced local heating of the central electrode which produces the initial working vapor (copper, see figure 11(b)) in which the microdischarges are established [23-25]. In this case, the situation is describable as cathode spots emitting metal vapor into the liquid water. Apparently these discharges invariably heat the water locally to vapor thereby making the transition to predominately water vapor. This phenomenological model is supported by the observation that the discharge initially starts as a greenish glow indicating electrode metal (copper) vaporization due to localized field emission heating of some protrusion on the electrode. This notion is also supported by spectra acquired from the source as shown in figure 12. Here in addition to the prominent $\mathrm{OH}$ band around $308 \mathrm{~nm}$, the hydrogen $656 \mathrm{~nm}$ Balmer line, and a small peak at $777 \mathrm{~nm}$ corresponding to oxygen, copper 324 and $328 \mathrm{~nm}$ lines are also apparent.

The water heating is also fairly rapid in this operating mode. The heating is also quite nonuniform with the upper portion heating rapidly and the lower portion remaining nearly at room temperature over the test duration. The water heating is assumed to be due to heat transfer between the steam bubbles and the water as buoyant forces lift the bubbles toward the surface. Heating rates and behavior with and without airflow are shown in figure 8 . As can be seen here, the heating rate is actually somewhat higher for the zero airflow case. The fact that the heating rates without airflow is more effective at heating is not surprising. In the zero airflow case, the steam is actually produced directly by the discharge, thereby allowing direct interaction between steam and water. With airflow, heating relies on energy transfer between the discharge and cooler, injected air, which in turn heats the water. Figure 13 illustrates waveforms for the cases with and without airflow ( $4 \mathrm{kHz}$ operation). As can be seen in the figure, the waveforms have similar general shape and behavior, suggesting that similar processes are driving the discharges, with the primary difference being that the working gas in the zero-flow case is produced at the source itself.
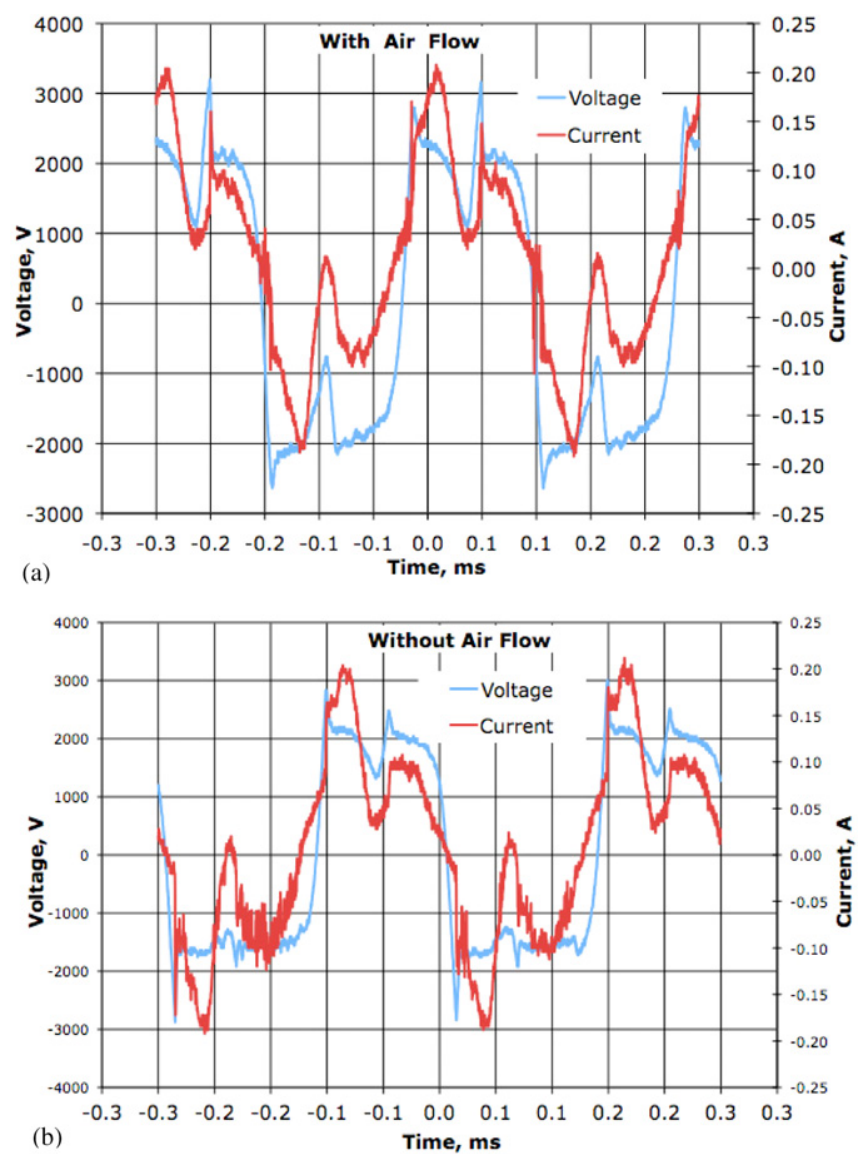

Figure 13. $I V$ characteristics of the plasma jet at $4 \mathrm{kHz}$ with $(a)$ and without airflow $(b)$.

\subsection{MB dyed water response to plasma treatment}

Anecdotal evidence of water chemical effects induced by the plasma jet was documented using $\mathrm{MB}$, an oxidation indicator. $\mathrm{MB}$ has been used in the past to test for chemical reactivity in both purely chemical and plasmaassisted chemistry studies $[8,26,27]$. The MB solution turns clear in a reducing environment. In this work, a sample of $0.0046 \mathrm{~g}$ of MB was mixed into a $60 \mathrm{ml}$ of dionized water. This sample was then exposed to the air discharge plasma ( $1 \mathrm{kHz}$ ) cyclically for approximately $8 \mathrm{~min}$. The cyclic (on/off) exposure was necessary to prevent boiling. An identical, MB-water sample was also processed by simple boiling for about $8 \mathrm{~min}$ using a hot plate. Three samples were compared: (1) untreated (2) plasma-treated (3) boil-treated. Figure 14 portrays typical results of the effect that the plasma treatment had on the dye-laden water. A visual assessment revealed essentially no difference between the boiled and control samples while the plasma-treated samples were observed to be nearly clear solutions. The lack of difference between the control and the boiled samples suggests that the clearing of the solution for the plasma-treated sample was not thermal, rather chemical in nature.

A Baush and Lomb Spectronic 20 was used to determine the transmittance of light through the plasma-treated sample relative to that of deionized water. The spectrophotometer was set at a wavelength of $609 \mathrm{~nm}$, the location of the peak 


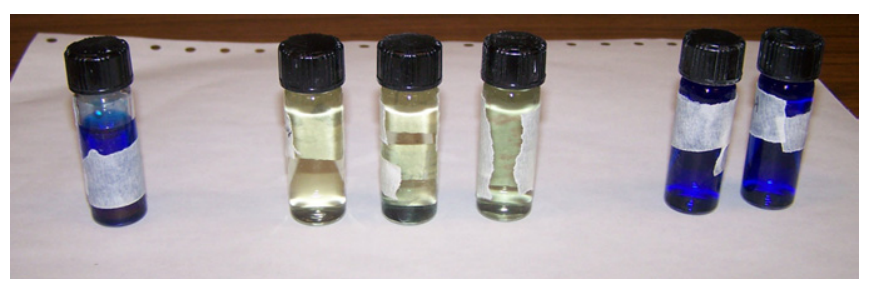

Figure 14. Comparison between untreated (leftmost),

plasma-treated (middle) and boiled-treated (rightmost) MB samples. (Discharge: $1 \mathrm{kHz}, 2500 \mathrm{~V}$ amplitude, $1.9 \mathrm{~L} \mathrm{~min}^{-1}$.)

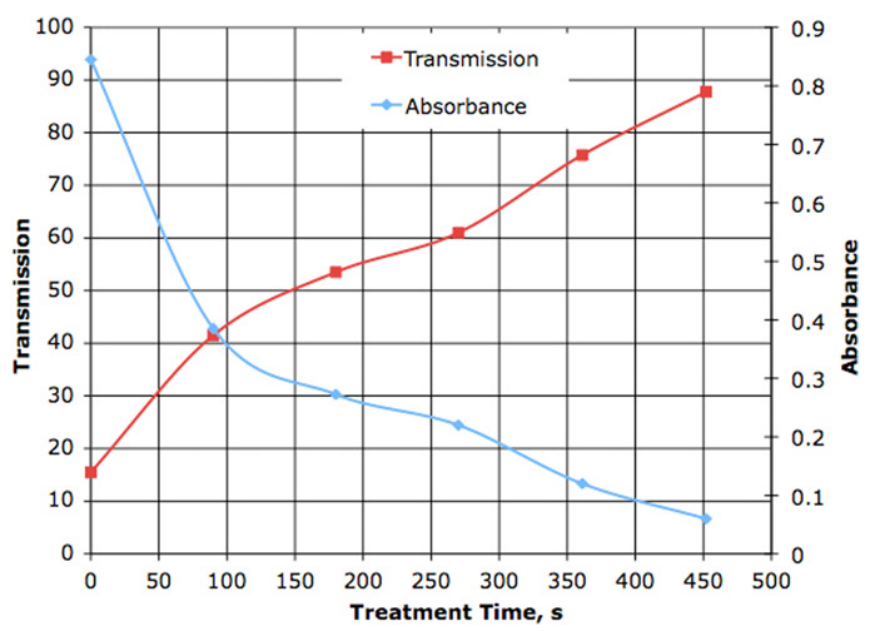

Figure 15. Variation in the transmission and absorbance at $609 \mathrm{~nm}$ of a MB-dionized water solution as a function of plasma treatment time. (Discharge: $1 \mathrm{kHz}, 2500 \mathrm{~V}$ amplitude, $1.9 \mathrm{~L} \mathrm{~min}^{-1}$.)

absorption band of MB. The spectrophotometer indicated just over $70 \%$ transmittance after the $8 \mathrm{~min}$ plasma treatment. To obtain insight into the variation in the transmittance as a function of treatment time, a slightly more dilute MB-deionized water mixture was used $(0.0043 \mathrm{ml}$ of MB was diluted into $100 \mathrm{ml}$ of water). This allowed for assessment of transmissivity of samples before plasma treatment. Samples were extracted periodically over the treatment period to map out the variation in the MB concentration with time. The variation in the transmittance and absorbance of the treated MB solution as a function of time is depicted in figure 15, which indicates a monotonic increase in transmission with treatment time. Again as can be seen here, significant changes in the MB concentration is brought upon by the plasma treatment.

\section{Conclusions}

The operation of a dielectric barrier discharge plasma jet in water was characterized. The discharge was observed to evolve from a localized microdischarge to an arc-like discharge with increasing voltage. The water heating rate and the water-power absorption fraction driven by the plasma jet was found to be nearly independent of the excitation frequency. Discharge operation without airflow was also demonstrated. In this case, the water heating mechanism appears to be heat transfer between the self-produced steam bubbles and the ambient water. Calorimetry estimates suggest the good heat transfer between plasma and water with water-power absorption fractions as high as $60 \%$ with the remaining discharge power being consumed by ionization, excitation, radiation and electrode heating. Emission spectra revealed significant amounts of excited nitrogen and $\mathrm{OH}$ with airflow and essentially $\mathrm{OH}$ and hydrogen without airflow, suggesting the presence of active species that could modify water chemistry. The presence of copper in the measured emission spectra suggested that electrode processes play a key role in the maintenance of the arc-like mode. Water chemistry modification of MB-laden water due to plasma jet action was inferred through the observation of water clearing after plasma jet treatment.

\section{References}

[1] Eliasson B and Kogelschatz U 1991 IEEE Trans. Plasma Sci. 191063

[2] Cabane B and Vuilleumier R 2005 C. R. Geosci. 337159

[3] Gao J et al 2001 Thin Solid Films 390154

[4] Malik M, Ghaffar A and Malik S 2001 Plasma Sources Sci. Technol. 1082

[5] Banat I, Nigam P, Signh D and Marchant R 1996 Bioresource Technol. $\mathbf{5 8} 217$

[6] Anppilov A et al 2001 J. Phys. D: Appl. Phys. 34993

[7] Sunka P 2001 Phys. Plasmas 82587

[8] Sugiarto A et al 2003 J. Electrostat. 58135

[9] Gao J et al 2001 Plasma Sci. Technol. 3641

[10] Schutze A et al 1998 IEEE Trans. Plasma Sci. 461685

[11] Laroussi M and Akan T 2007 Plasma Process. Polym. 4777

[12] Schutze A et al 1998 IEEE Trans. Plasma Sci. 261685

[13] Kogelschatz 2003 Plasma Chem. Plasma Process. 231

[14] Kogelschartz U 2002 IEEE Trans. Plasma Sci. 301400

[15] Okazaki S et al 1993 J. Phys. D: Appl. Phys. 26889

[16] Shi J J et al 2003 J. Appl. Phys. 946303

[17] Nie Q et al 2007 Appl. Phys. Lett. 90221504

[18] Park J 2001 J. Appl. Phys. 89 20-8

[19] Feng R, Castle G S P and Jayaram S 1998 IEEE Trans. Indust. Appl. 34563

[20] Manley T C 1943 Trans. Electrochem. Soc. 8483

[21] Pearse R W B 1950 The Identification of Molecular Spectra (New York: Wiley)

[22] Guerra-Mutis M H et al 2003 Plasma Sources Sci. Technol. 12165

[23] Akiyama H 2000 IEEE Trans. Dielectr. Electr. Insul. 7646

[24] Lisitsyn I V et al 1999 IEEE Trans. Dielectr. Electr. Insul. $6351-6$

[25] Jones H M and Kunhardt E E 1994 IEEE Trans. Dielectr. Electr. Insul. 11016

[26] Snehalatha T, Rajanna K C and Saiprakash P K 1997 J. Chem. Edn 74228

[27] Iishijima T et al 2007 Appl. Phys. Lett. 91121501 